\title{
Laparoscopic sentinel lymph node mapping after cervical injection of indocyanine green for endometrial cancer - preliminary report
}

\author{
Ewa Surynt ${ }^{1}$, Malgorzata Reinholz-Jaskolska ${ }^{1}$, Mariusz Bidzinski ${ }^{1,2}$ \\ ${ }^{1}$ Department of Gynecology, 'Inflancka' Hospital, Warsaw, Poland \\ ${ }^{2}$ Department of Health Science, Jan Kochanowski University, Kielce, Poland
}

Videosurgery Miniinv 2015; 10 (3): 406-412

DOI: 10.5114/wiitm.2015.54099

\begin{abstract}
Introduction: Endometrial cancer (EC) has an increasing incidence worldwide, with lymph node metastases as the main prognostic factor. Systemic lymphadenectomy is connected with elevated morbidity. Sentinel lymph node (SLN) biopsy is intended to avoid extensive lymphadenectomy and provide significant oncologic information.

Aim: To evaluate the accuracy of laparoscopic SLN biopsy guided by indocyanine green (ICG) injection into the cervix in EC patients and to develop ideas to improve this method. The optimal time from dye injection to lymph node visualization was assessed.

Material and methods: This retrospective study was conducted between July 2014 and March 2015 in a group of 9 women with EC, at low and intermediate risk of recurrence, scheduled for total laparoscopic hysterectomy and pelvic lymphadenectomy. All patients underwent cervical ICG injection and SLN biopsy, followed by surgery. Pelvic lymph nodes were located using an ICG endoscopic camera.

Results: The following data were collected: There were 9 patients with endometrial cancer at low and intermediate risk of recurrence. Median patient age was 59 years, median body mass index (BMI) $28 \mathrm{~kg} / \mathrm{m}^{2}$, endometrioid adenocarcinoma in 9 cases, grading: G1 - 1 patient, G2 - 8 patients. No intraoperative or postoperative complications were noted. Median time from ICG injection and SLN detection during surgery was $25 \mathrm{~min}$. There were no lymph nodes metastasis, all identified by the SLN protocol using ICG injection.

Conclusions: Sentinel lymph node mapping can play a significant role in lymph node assessment and staging in early-stage EC patients with low risk of recurrence according to the ESMO classification. The use of SLN mapping in $E C$ is much needed and the therapeutic benefit is high.
\end{abstract}

Key words: sentinel lymph node, endometrial cancer, indocyanine green (ICG), total laparoscopic hysterectomy.

\section{Introduction}

The rate of endometrial cancer (EC) is steadily increasing. Most patients present with early-stage disease, and the incidence of metastases in this group is low. However, standard treatment includes a complete or selective pelvic and para-aortic lymphadenectomy, with surgical staging as the most impor- tant prognostic factor. Recent studies demonstrated no therapeutic benefit of complete lymphadenectomy in patients with early-stage EC [1]. Additionally, early-stage EC corresponds to low and intermediate risk, according to the European Society of Medical Oncology (ESMO) [1]. In contrast, patients in highrisk ESMO groups (type 1 , grade 3 endometrioid car- 
cinoma with myometrial infiltration over 50\%, type 2 EC I Endometrial serous and clear cell carcinoma) need staging including pelvic and para-aortic lymphadenectomy.

Sentinel lymph node (SLN) mapping, which has been used in breast cancer or skin melanoma, might be an acceptable surgical strategy between radical lymphadenectomy and no lymphadenectomy in patients with endometrial cancer. Sentinel lymph node mapping is based on the idea that lymph node metastases occur following lymph drains from the tumor [2]. There is a spread of tumor cells to the first lymphatic station in SLN. This node should limit further distribution of tumor cells. The nodes after SLN should also be negative. Sentinel lymph node allows one both to avoid lymphadenectomy complications and to establish proper EC staging.

Although the definition of the sentinel node was presented by Cabanas in 1977, the idea of the sentinel node had been earlier described by Braithwaite in 1923, and the term 'the sentinel node' was first used by Gould in 1960 [3, 4]. Dynamic development of the sentinel node biopsy technique began in 1992, when Morton et al. published their report on the use of this method in patients with skin melanoma [4].

The intention of SLN mapping is to avoid overstaging and overtreatment by performing complete lymphadenectomy of the negative nodes and pathologic focusing on identification of micrometastases. Radical lymph node dissection may cause side effects, e.g. leg lymphedema and lymphocysts, which can have a negative influence on the patient's quality of life. Accurate surgical staging and lymph node dissection constitute the most important prognostic factors. To the best of our knowledge, there have been no prospective randomized trials assessing the efficacy of SLN mapping in EC, and no long-term survival data are available [2]. There are a couple of SLN mapping techniques. A radioactive tracer and color dye (blue or green) are used to locate the 'hot' nodes or visualize colored lymph nodes [2]. Sentinel lymph nodes are assessed as positive if they contain macrometastases (tumor $>2 \mathrm{~cm}$ ), micrometastases (tumor $0.2-2.0 \mathrm{~mm}$ ), or isolated tumor cells (single tumor cells $\leq 0.2 \mathrm{~mm}$ ). Based on the place of injection, three different types of SLN mapping techniques were proposed: uterine subserosal, cervical, and endometrial during hysteroscopy [2]. We are of the opinion that the cervical injection is the most appropriate because the lymphatic drainage to the uterus is from the parametria, which is why a superficial and deep cervical injection seems to be better. The cervix is easily accessible and, in the case of EC, rarely changed by anatomical variations. Furthermore, the cervix in EC patients is seldom scarred from previous surgical procedures. The main argument against the cervical injection is a lower para-aortic detection rate. However, when pelvic nodes are negative, metastases are unlikely to be found in the para-aortic nodes [5]. Additionally, there is no definitive well-documented relationship between para-aortic nodal assessment and improved overall survival (OS) [6]. Kang et al. reported a decrease in detection rates when the cervical injection method was not used, although without statistical significance. These authors recommended avoiding subserosal injection only because of its decreased sensitivity [7]. Based on their experience, the cervical-only approach is recommended.

\section{Aim}

The aim of our study was to assess the accuracy of laparoscopic SLN biopsy performed with cervical ICG navigation. It is our intention to evaluate SLN detection rate, sensitivity, specificity and false-negative rates of the method, considering the final pathological result of complete lymphadenectomy in the future. Additionally, the optimal time from dye injection to lymph node visualization was assessed.

\section{Material and methods}

We conducted a retrospective study from July 2014 to March 2015 in 9 women (1 nullipara and 8 multiparas) with EC at low and intermediate risk of recurrence, scheduled for total laparoscopic hysterectomy (TLH), bilateral salpingo-oophorectomy and pelvic lymphadenectomy. The inclusion criteria were as follows: histologically confirmed EC, no evidence of extrapelvic metastases on initial imaging staging (abdomen and pelvis computed tomographic scan). The exclusion criteria were as follows: lack of written informed consent, contraindication to laparoscopy, evidence of peritoneal or distant metastasis detected before or during surgery, conversion to laparotomy. All patients underwent cervical ICG injection and SLN biopsy, followed by TLH, bilateral salpingo-oophorectomy, and pelvic lymphadenectomy. The following data were collected: median patient age, 
median body mass index (BMI), histologic type and grading. Estimated median blood loss, median laparoscopy time, and intraoperative and postoperative complications were analyzed intraoperatively.

FIGO staging was based on histopathology. Sentinel lymph node detection during the operation was analyzed (median time from ICG injection into the cervix and its detection during surgery, bilaterally or unilaterally).

The SLN biopsy technique was as follows: before the surgery the patient was placed in a lithotomy position and ICG-PULSION (indocyanine green) was injected into the cervix with the 4-quadrant option at the noted time, i.e. approximately $15 \mathrm{~min}$ before placement of the trocars. Then, the patient underwent laparoscopic surgery as standardized by FIGO 2009 [8].

The operative technique was as follows: the patient was routinely positioned in the semi-lithotomy position, prepared and draped in a sterile fashion, then the uterine Hohl manipulator and 4 laparoscopic trocars were placed. One trocar was inserted at the level of the umbilicus, holding the camera and optics, then a 5-mm trocar and a 10-mm trocar were introduced in each lower part of the pelvis and about $3 \mathrm{~cm}$ below the umbilicus and lateral to the epigastric vessels. Inspection of the abdominal cavity constituted the first part of the procedure. Pelvic lymph nodes were located using an ICG endoscopic camera (Storz). After locating SLNs, each node was separately removed. Hysterectomy was followed by complete bilateral pelvic lymphadenectomy. A unilateral SLN was found in one case.

The lymph nodes were analyzed with frozen sections. No metastatic disease was detected in SLNs in frozen and in routine (hematoxylin-eosin) sections. The tumor was histologically classified and staged using the 2009 FIGO classification [9]. The false negative rate was defined as the number of procedures with negative SLN divided by the number of procedures in which SLN was recognized in the final micro- scopic examination of SLN or a positive lymph node was found in the removed iliac-obturator nodes [10].

\section{Results}

The following data were collected: 9 patients with endometrial cancer at low or intermediate risk of recurrence. Demographic and histopathology data are presented in Table I. Clinical and complication data are presented in Table II.

Sentinel lymph nodes were detected during the operation: bilaterally in $8(88.9 \%)$ cases and unilaterally (right side) in 1 (11.1\%) case. Median time from ICG injection into the cervix and SLN detection during surgery was 25 min (range: 20-30 min).

There were no positive lymph nodes, no metastasis were recognised, all detected by the SLN protocol using the ICG injection.

We also identified a correlation between negative SLNs and uninvolved pelvic non-SLNs (Table III).

\section{Discussion}

Since incorporating the SLN mapping algorithm in 2008 , the rate of complete lymphadenectomy has decreased from $65 \%$ to $23 \%$ [2]. The median number of removed nodes decreased from 20 to 7 [2]. Ten or more lymph nodes were required for proper surgical staging, not SLN mapping. The aim of SLN mapping is to reduce the number of nodes needed to be removed for staging and to avoid more extensive dissections [2].

Three methods of SLN mapping have been recommended: use of technetium-99 ( ${ }^{99 \mathrm{~m} T c)}$ ), blue dye and ICG. One of these methods used to identify SLN is lymphoscintigraphy. A radiolabeled colloid, usual-

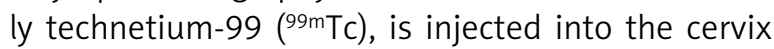
one day before or on the day of the operation. The technetium is carried via lymph through lymphatic vessels to SLNs. The preoperative lymphoscintigram is taken approximately 20-30 min after the injection

Table I. Demographic and histological data

\begin{tabular}{|lcccc|}
\hline Parameter & Min. & Max. & Mean & No. \\
\hline Age $[$ years] & 26 & 67 & 59 & \\
\hline BMI $\left[\mathrm{kg} / \mathrm{m}^{2}\right]$ & 19 & 35 & 28 & 1 \\
\hline Adenocarcinoma G1 & & & & 8 \\
\hline Adenocarcinoma G2 & & & & \\
\hline
\end{tabular}


Table II. Clinical and complication data

\begin{tabular}{|c|c|c|c|c|}
\hline Parameter & Min. & Max. & Mean & No. \\
\hline \multicolumn{5}{|l|}{ FIGO stage: } \\
\hline la & & & & 3 \\
\hline $\mathrm{lb}$ & & & & 4 \\
\hline$\| a$ & & & & 1 \\
\hline IIla & & & & 1 \\
\hline Blood loss [ml] & 200 & 300 & 270 & \\
\hline Operative time [min] & 90 & 210 & 130 & \\
\hline \multicolumn{5}{|l|}{ SLN localization: } \\
\hline Bilateral & & & & 8 \\
\hline Unilateral & & & & 1 \\
\hline Time to SLN localization [min] & 20 & 30 & 25 & \\
\hline Lymphorrhea & & & & 2 \\
\hline Obturator nerve irritation & & & & 1 \\
\hline
\end{tabular}

Table III. Patient characteristics by presence of nodal metastasis

\begin{tabular}{|lcccc|}
\hline Variable & No. & Min. & Max. & Mean \\
\hline Number of removed SLNs (8 cases) & 59 & 1 & 15 & 5 \\
\hline Number of removed pelvic nodes & 160 & 4 & 25 & - \\
\hline Number of involved SLNs & 0 & - & - & \\
\hline Metastases on non-SLNs & 0 & - & - \\
\hline
\end{tabular}

to locate the SLNs. Gamma probes are used to detect the 'hot' nodes. Gamma probes can be used laparoscopically or as a handheld open-surgery probe [2]. Single-photon emission computed tomography (SPECT), which uses 3-dimensional localization of the 'hot' nodes, also detected through a gamma probe, can be used [8].

Colored dye injection is used to identify SLN as well. Blue dye (isosulfan blue 1, methylene blue 1\%, patent blue $2.5 \%$ sodium) is injected at the beginning of the operation, under anesthesia. The dye is injected slowly, similarly to the radiotracer into the cervical submucosa and stroma, into 4 quadrants of the cervix [2]. The complication rate is low; mostly allergic reactions (edema, pruritus, respiratory distress and shock) have been observed. The urine can be green for up to $24 \mathrm{~h} \mathrm{[2]}$.

Fluorescent SLN imaging with green dye (indocyanine green (ICG)) is believed to be an excellent dye for SLN mapping. The method of injection is similar to blue dye. lodine allergy constitutes the main contraindication. Fluorescent SLN identification with ICG is the preferred mapping approach [2].

The SLN surgical algorithm includes peritoneal and serosal evaluation, retroperitoneal evaluation, including dissection of all SLNs and any suspicious nodes, and finally pelvic, common iliac and interiliac lymph node dissection, if there is no mapping on the pelvis [2].

Nejc et al. have described lymphoscintigraphy, radiation dosage, and the problem of medical staff safety in surgery for breast cancer and skin melanoma [4, 11, 12]. They analyzed the radiation dosage on the hands of the surgeon, anesthesiologist, assistant surgeon, and nurses [4, 11, 12]. According to the International Commission on Radiological Protection, the main surgeon is at the highest risk and is allowed to perform 2150 melanoma opera- 
tions and 2250 procedures for breast cancer per year maximally $[4,11,12]$. Summing up, lymphoscintigraphy radiation is a safe method, but requires special equipment and medical staff, in contrast to ICG injection. It can be used only in specialist medical centers, but most EC patients are treated outside of comprehensive oncological hospitals.

Disadvantages of lymphoscintigraphy include radiocolloid injection prior to anesthesia and surgery, but ICG injection is given intraoperatively.

There are three different types of SLN mapping techniques, based on the place of injection: uterine subserosal, cervical, and endometrial via hysteroscopy [2]. Cervical ICG injection achieves a higher SLN detection rate and a similar anatomic nodal distribution as hysteroscopic endometrial injection for SLN mapping in patients with endometrial cancer [13]. Abu-Rustum et al. prefer the cervical injection, whereas other authors claim that peritumoral or hysteroscopic injection is more appropriate $[14,15]$.

Sinno et al. identified SLN using cervical ICG injection bilaterally (78.9\%) [16]. Five women (7\%) had positive lymph nodes; all were identified by the SLN protocol [16].

Early-stage EC with low and intermediate ESMO risk groups requires pelvic lymphadenectomy [1]. In contrast, patients in the ESMO high risk group (type 1, grade 3 endometrioid carcinoma with myometrial infiltration over 50\%, type 2 EC Endometrial serous and clear cell carcinoma) require staging including pelvic and para-aortic lymph node dissection [17]. The SEPAL study demonstrated that systemic pelvic and para-aortic lymphadenectomy for patients in the high risk group was associated with increased overall and disease-free survival [18]. Kumar et al. reported that pelvic and para-aortic lymph node metastases were observed in $17 \%$ and $9 \%$ of the analyzed patients [19]. They suggested that these patients do not benefit from lymphadenectomy; instead it exposes them to the risk of postoperative complications and impaired quality of life [1].

On the other hand, previous studies on early-stage EC demonstrated the relevance of sentinel lymph node biopsy in detection of lymph node metastases in low and intermediate ESMO risk groups [14]. In these cases, SLN biopsy is a compromise between systemic and no lymphadenectomy [20].

The question whether patients classified as low and intermediate risk should be excluded from the SLN procedure remains a separate issue. Systemic pelvic lymphadenectomy is recommended for these patients, but in high-risk groups more than three quarters had no para-aortic lymph node metastases. Abu-Rustum et al. report that the risk of 'skip' metastases in case of negative pelvic SLN is about 2\% [5]. This strategy should be carefully considered because of the risk of intra- and post-operative complications linked directly to para-aortic lymphadenectomy [21, 22]. Additionally, the incidence of para-aortic lymph node metastases is relatively low $[5,22]$.

According to two other randomized trials, there is no advantage in performing systemic lymphadenectomy in patients with early-stage EC [23-27]. The debate whether lymphadenectomy should be the standard EC management continues. Darai et al. demonstrated that SLN biopsy predicts lymph node status for patients with early-stage EC and that lymph node involvement was more accurately detected by ultrastaging [28]. Unfortunately, we had no possibility of performing pathologic ultrastaging.

In the report by Darai et al., patients with positive SLN underwent immediate para-aortic lymphadenectomy (PAL) and adjuvant whole pelvic external beam radiotherapy (EBRT) (50 Gy in 25 fractions) and chemotherapy more frequently, while no difference in recurrence-free survival (RFS) according to the SLN status was observed [28]. Additionally, the same study proved that there is the coincidence of negative SLN on surgical management with indications for adjuvant therapies [28].

Naoura et al. presented results indicating that SLN biopsy associated with lymphovascular space invasion (LVSI) status can detect which high-risk patients with EC, excluding the low and intermediate risk of recurrence group, would benefit from comprehensive staging [29]. They focused, similarly to Darai et al. [28], on ultrastaging detecting metastases undiagnosed by conventional histology in $41 \%$ of the patients [28, 29]. Preoperative findings classified 146 patients in the Naoura study as ESMO low or intermediate risk (81\%) and 34 as high risk (19\%) [29]; $29 \%$ of the patients in the presumed high-risk group were down-staged on final histology and $28 \%$ of the women initially diagnosed with type 2 were finally classified as 'type $1 \mathrm{EC}$ ' [29]. That study confirmed a high detection rate of SLN in patients with EC, even for those of the high-risk ESMO group [29]. Regardless of whether the SLN detection rate and bilateral detection were high in both low/intermediate and high-risk groups, the false negative rate 
was lower for patients with low/intermediate EC [29]. These results call into question the usefulness of the SLN procedure in high-risk groups of patients [29]. Their report showed that the SLN procedure is crucial for patients with early-stage EC. Moreover, in patients with an indication for a second surgical procedure, the presence of LVSI may help select patients who would benefit from lymph node dissection [29].

\section{Conclusions}

Our preliminary study confirms the usefulness of the ICG technique for SLN identification. Future investigations should concentrate on finding the best place for tracer injection and estimation of accuracy of the presented diagnostic method. Additionally, further studies should focus not only on the SLN biopsy status but also on stratifying adjuvant therapies according to the SLN results.

Our preliminary observations provide some information about the usefulness of the ICG method in definition of SLN in endometrial cancer patients. The SLN was detected and no false negative results were observed in all of the analyzed cases. Median time from ICG injection to lymph node visualization was 25 min. The acceptability of the method was high, among both patients and staff.

\section{Conflict of interest}

The authors declare no conflict of interest.

\section{References}

1. May K, Bryant A, Dickinson HO, et al. Lymphadenectomy for the management of endometrial cancer. Cochrane Database Syst Rev 2010; 1: CD007585.

2. Abu-Rustum NR. Sentinel lymph node mapping for endometrial cancer: a modern approach to surgical staging. I Natl Compr Canc Netw 2014; 12: 288-97.

3. Gould EA, Winship T, Philbin PH, et al. Observation on a "sentinel node" on cancer of the parotid. Cancer 1960; 13: 77-8.

4. Nejc D, Jeziorski A. Sentinel node biopsy - a summary of own experiences. Nowotwory J Oncol 2008; 58: 405-12.

5. Abu-Rustum NR, Gomez JD, Alektiar KM, et al. The incidence of isolated para-aortic nodal metastasis in surgically staged endometrial cancer patients with negative pelvic lymph nodes. Gynecol Oncol 2009; 115: 236-8.

6. Barlin JN, Zhou Q, St Clair CM, et al. Classification and regression tree (CART) analysis of endometrial carcinoma: seeing the forest for the trees. Gynecol Oncol 2013; 130: 452-6.

7. Kang S, Yoo HJ, Hwang LH, et al. sentinel lymph node biopsy in endometrial cancer: meta-analysis of 26 studies. Gynecol Oncol 2011; 123: 522-7.
8. Pandit-Taskar N, Gemingnani ML, Lall A, et al. Single photon emission computed tomography SPECT-CT improves sentinel node detection and localization in cervical and uterine malignancy. Gynecol Oncol 2010; 117: 59-64.

9. Pecorelli S. Revised FIGO staging for carcinoma of the vulva, cervix and endometrium. Int J Gynaecol Obstet 2009; 105: 103-4.

10. Favero G, Pfifer T, Ribeiro A, et al. Laparoscopic sentinel lymph node detection after hysteroscopic injection of Technetium-99 in patients with endometrial cancer. Int J Gynecol Cancer 2015; 25: 423-30.

11. Nejc D, Wrzesiński M, Piekarski J, et al. Sentinel node biopsy in skin melanoma patients - measurements of absorbed doses of radiation to the hands of medical staff. J Surg Oncol 2006; 93: 355-61.

12. Nejc D, Wrzesiński M, Piekarski J, et al. Sentinel node biopsy in patients with breast cancer - evaluation of exposure to radiation of medical staff. Eur J Surg Oncol 2006; 32: 133-8.

13. Rossi EC, Jackson A, Ivanova A, et al. Detection of sentinel nodes for endometrial cancer with robotic assisted fluorescence imaging: cervical versus hysteroscopic injection. Int J Gynecol Cancer 2013; 23: 704-11.

14. Khoury-Collado F, Abu-Rustum NR. Lymphatic mapping in endometrial cancer: a literature review of current techniques and results. Int J Gynecol Cancer 2008; 18: 1163-8.

15. Abu-Rustum NR, Khoury-Collado F, Gemignami ML. Techniques of sentinel lymph node identification for early-stage cervical and uterine cancer. Gynecol Oncol 2008; 111: S44-50.

16. Sinno AK, Fader AN, Roche KL, et al. A comparison of colorimetric versus fluorometric sentinel lymph node mapping during robotic surgery for endometrial cancer. Gynecol Oncol 2014; 134: 281-6.

17. Colombo N, Preti E, Landoni F, et al. Endometrial cancer: ESMO Clinical Practice Guideliness for diagnosis, treatment and follow-up. J Eur Soc Med Oncol 2011; 22 (suppl. 6): vi35-9.

18. Todo Y, Kato H, Kaneuchi M, et al. Survival effect of para-aortic lymphadenectomy in endometrial cancer (SEPAL study): a retrospective cohort analysis. Lancet 2010; 375: 1165-72.

19. Kumar S, Podratz KC, Bakkum-Gamez JN, et al. Prospective assessment of the prevalence of pelvic, para-aortic and high para-aortic lymph node metastasis in endometrial cancer. Gynecol Oncol 2014; 132: 38-43.

20. Kitchener H, Swart AMC, Qian Q, et al. Efficacy of systematic pelvic lymphadenectomy in endometrial cancer (MRC ASTEC trial): a randomized study. Lancet 2009; 373: 125-36.

21. Achouri A, Huchon C, Bats AS, et al. Complications of lymphadenectomy for gynecologic cancer. Eur J Surg Oncol 2013; 39: 81-6.

22. Naoura I, Canlorbe G, Bendifallah S, et al. Relevance of sentinel lymph node procedure for patients with high-risk endometrial cancer. Gynecol Oncol 2015; 136: 60-4.

23. ASTEC study group, Kitchener H, Swart AMC, Qian Q, et al. Efficacy of systemic pelvic lymphadenectomy in endometrial cancer (MRC ASTEC trial): a randomized study. Lancet 2009; 373: 125-36.

24. May K, Dickinson HO, Kehoe S, et al. Lymphadenectomy for the management of endometrial cancer. Cochrane Database Syst Rev 2010; 1: CD007585. 
25. Benedetti Panici P, Basile S, Maneschi F, et al. Systemic pelvic lymphadenectomy vs. no lymphadenectomy in early-stage endometrial carcinoma: randomized clinical trial. J Natl Cancer Inst 2008; 100: 1707-16.

26. Ballester M, Dubernard G, Lecuru F, et al. Detection rate and diagnostic accuracy of sentinel-node biopsy in early-stage endometrial cancer: a prospective multi-center study (SENTI-ENDO). Lancet Oncol 2011; 12: 469-76.

27. Kitchener HC. Sentinel-node biopsy in endometrial cancer: a win-win scenario? Lancet Oncol 2011; 12: 413-4.

28. Darai E, Dubernard G, Bats AS, et al. Sentinel node biopsy for the management of early stage endometrial cancer: long-term results of the SENTI-ENDO study. Gynecol Oncology 2015; 136: 54-9.

29. Naoura I, Canlorbe G, Bendifallah S, et al. Relevance of sentinel lymph node procedure for patients with high-risk endometrial cancer. Gynecol Oncology 2015; 136: 60-4.

Received: 23.06.2015, accepted: 19.08.2015. 